\title{
Neural activity in the lower pathway of the somatosensory system in the presence of silicon interfaces
}

\author{
F. Panetsos, V. Bonacasa, V.A. Makarov \\ Department of Applied Mathematics, School of Optics, Complutense University of Madrid, Madrid, Spain
}

\begin{abstract}
In this communication, we present results of experimental work carried out in the frame of the ROSANA project aiming at the investigation of the interactions between sensory inputs and the activity of Central Nervous System (CNS) neurons in the creation of the internal representations of real-world stimuli. We implanted sieve microelectrodes in the peripheral nerve of rats and we obtained functional regeneration of the sensory nerves. We recorded the electrical activity of the regenerated nerve fibers and also of the relay neurons of the first station of the somatosensory pathway. Finally we developed mathematical models of the oscillatory neurons involved in the information processing that fit well with our experimental data.
\end{abstract}

Keywords - Oscillations, signal processing, artificial prostheses, sieve microelectrodes, neural activity, somatosensory system

\section{INTRODUCTION}

The Central Nervous s System (CNS) receives a very large amount of information from the external world, coded and transmitted through the nerves as complex spatiotemporal and intensity patterns of electrical pulses and then analyzed and combined in subsequent processing stages to produce perception [1]. In a first moment, information is represented and conveyed along the neural membranes under the form of, initially passive and then active, electrical potentials (spikes) that are transmitted to some hundred or thousands of relay neurons. An important characteristic of this process is that information, is not only transmitted but also transformed. Most neurons do not differ significatively in their electrical properties and they carry out different functions because of the individual afferent and efferent connections they make [2]. That means that these neurons perform like dedicated processing devices whose output depends strongly on the spatiotemporal and intensity characteristics of the incoming signals. Then, the perception of the external world by the CNS is the result of a constant interplay between incoming signals and dynamic internal representations of the external world.

Consequently, if we are interested in the design of artificial devices to be directly connected to the peripheral nerves and devoted to transduce the physical characteristics of the external world in bioelectrical signals we have to take into account the coding procedures of the CNS, the lines along which the information is conveyed, as well as the interactions between incoming signals and intrinsic neural

Work supported by the EU (contracts IST-2001-34892 and IST-2001-34893) and the spanish FIS (grant 01/0822) activity of the relay cells. Since both, the output and the performances of the neurons in the different relay stations of the sensory pathways are severely affected if the incoming signals are not the expected ones, we have to generate signals that could be correctly interpreted and processed, and the only way to do that is by coding and transmitting sensory information in a manner similar to that used by the CNS.

It is long established that neurons encode incoming signals through a linear function between stimulus intensity and spiking frequency [2]. However, encoding is not performed only in such a simple and inefficient way. A great amount of recent data suggests that more complex and efficient procedures like synchronized activity, interspike intervals (high-order statistics) and oscillating behavior of neural cells play an important role in information processing, whether sensory, motor or mental [3]. Unfortunately, our knowledge on these procedures is too limited to allow us to define protocols of artificial stimulation of the sensory nerves that could be appropriately interpreted by the CNS. Consequently, to understand how the electrical activity of sensory neurons can be reliably processed to generate the desired perceptions and to understand the internal representation of real-world stimuli in biological systems is a key fundamental step to progress in this field.

The objectives of the present work, carried out in the frame of the ROSANA project aiming at the investigation of how the interactions between sensory inputs and the activity of CNS neurons are used for creating the internal representation of real-world stimuli, was to implant a sieve microelectrode in a sensory nerve of an experimental animal, achieve functional regeneration of such nerve, record the neural activity of the nerve fibers crossing the electrode and test the "normal" activity of the neurons in the first relay station of the somatosensory pathway [4].

\section{METHODOLOGY}

Our experiments, carried out in accordance with the European Communities Council Directives (86/609/EEC) have been performed in rats according to the following operational scheme:

1) Implants: We implanted a sieve microelectrode [5] in the sciatic nerve of a rat by cutting the nerve, inserting the microelectrode and allowing the fibers to regenerate through the holes of the chip. During the surgical approach, the animals were anesthetized with sodium pentobarbital (35 $\mathrm{mg} / \mathrm{kg}$ i.p.) and maintained under aseptic conditions. 
2) Regeneration test: We verified the functional regeneration of the sciatic nerve by testing the reflexes of the animal when it was stimulated in the digits of the hindpaw.

3) Recording procedure: We stimulated the skin of a digit using mechanical stimuli and we recorded the activity of small ensembles of nerve fibers which regenerated through the sieve holes and the activity in the gracilis nucleus by means extracellular single-unit recordings. The animals were anesthetized with urethane (1.6 g/ $\mathrm{kg}$ i.p.) and placed in a stereotaxic device.

4) Data analysis and modeling: We performed shape analysis of the recorded potentials and peristimulus and autocorrelation histograms of the evoked neural activity in the gracilis nucleus. Mathematical models have been studied both analytically and by means of numerical simulations.

\section{RESULTS}

\section{A. Fiber recordings}

We recorded compound potentials from the sieve microelectrode indicating that small amounts of fibers of the sciatic nerve regenerated through the holes. From the available data we cannot determine the number of functional fibers regenerated through each hole nor the type of sensory submodality they are serving because the noise, mainly due to the experimental procedures and the available hardware, was very high.

\section{$B$. Single- and multi-unit neuron recordings}

In addition to the recordings of the electrical activity of the sciatic nerve we performed a systematic control of the response properties of the neurons in the nucleus gracilis limiting our research to the neurons in the region of the clusters [6] (the region of the nucleus that receives the most of the primary afferent fibers and who is responsible for the process of the information coming from the skin of the digits) and, additionally, to the neurons that received direct input from the fibers regenerated through the sieve microelectrode. The recordings identified two classes of relay neurons that correspond to the two cell types characterized in previous works in the intact nucleus [7].

Most of the cells $(69 \%)$ had a very low spontaneous activity which in, approximately $50 \%$ of the cases displayed a low-frequency rhythmic temporal pattern, normally under stimulation of their receptive fields. The neurons belonging to the second class had a high spontaneous activity in addition to a high-frequency rhythmic temporal pattern that did not change under peripheral stimulation of the receptive fields. Multiunit activity recorded by low-impedance microelectrodes displayed similar characteristics to the single-unit recordings of the first type (Fig. 1).

\section{Mathematical models}

Interpretation of the neural activity and the interactions between incoming signals and oscillations of the neural membrane potential by only analyzing the spiking properties of the relay neurons is not possible, at least with the present knowledge on these processes [8]. For this reason we developed mathematical models of neurons involved into the neural circuits in order to study their dynamical behavior. We consider neurons of the nucleus gracilis interconnected in small circuits with delays in the transmission lines using a simple biophysically motivated pulse-coupled network model to mimic information processing. The model consists of two phase oscillators mutually coupled via spikes. Both neurons receive sequences of external stimuli consisting of at least two spikes. The results of the model fit with the experimental data of both intact and electrode-implanted animals (Fig. 2), however, the time delays in both cases are not experimentally tested.
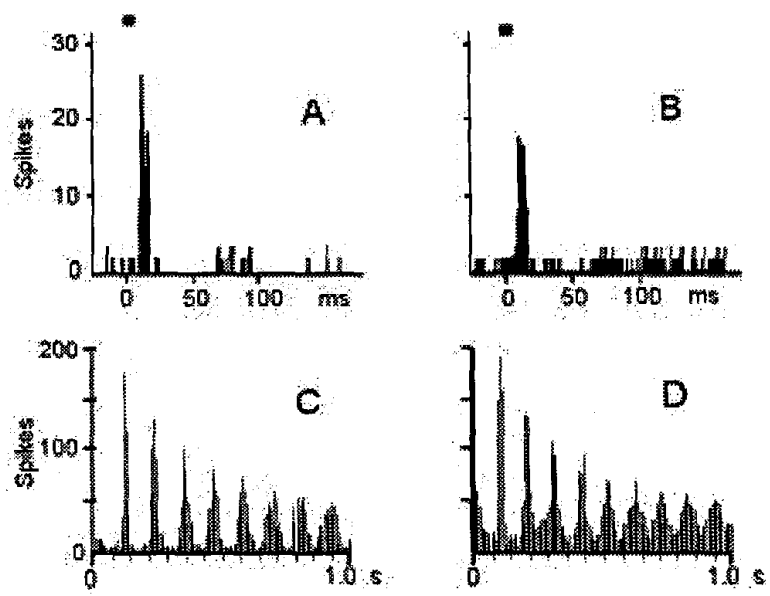

Fig. 1. Peristimulus histogram of a slowly oscillating (A) and a rapidly oscillating (B) neuron. Histograms calculated with 20 stimuli, upper lines indicate the duration of the stimuli. Autocorrelation histogram of the activity of a rapidly oscillating neuron at rest $(\mathrm{C})$ or under stimulation of its receptive field (D)

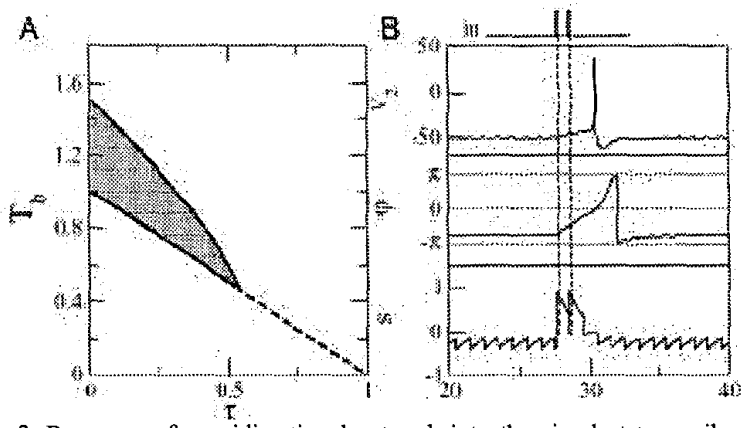

Fig. 2. Response of a unidirectional network into the simplest two-spikes stimulus with different timings when the stimulus enters to the projection neuron only. A). The domain of stimulus timings (relative delay $\tau$, interspike interval $T_{b}$ and inhibitory spike period $T_{f}$ ) where the projecting neuron overcomes threshold and fires a spike (dotted region). Otherwise the projecting neuron is silent. Dashed straight line corresponds to $T_{b}+\tau=T_{f}$. B). An example of traces from the projecting neuron when stimulus timings 
belongs to the domain shown in (A) $\left(\tau=0.2, T_{b}=0.83\right)$. Two vertical dashed straight lines show instants when stimulus spikes arrive to neuron "2". The inhibitory neuron fires tonic spikes with the period $T_{f}=1$.

\section{DISCUSSION}

Our experiments and the results we obtained suggest that we could achieve a good functional regeneration of the peripheral nerves through sieve microelectrodes with a real possibility to record small numbers of neural fibers. Moreover correlations between external stimuli, electrical potentials of the fibers and spiking activity of the relay neurons seems to be "easy" to achieve, at least when a single neuron is recorded. Complications will arise when simultaneous recordings from the sieve microelectrode and multielectrode recordings form the nucleus gracilis (or even the thalamus or cortex) will be attempted. Additional problems arise from purely anatomophysiological principles: if artificial sensors are to be directly connected to the peripheral nerve(s), only a very low number of fibers will be interfaced and, consequently, the study of direct fiber-neuron interactions is not sufficient to determine which information has to be coded and transmitted to the CNS. Moreover, by interfacing artificial sensors to sensory nerve will submit to each axon information concerning different sensory submodalities in an entirely unpredictable fashion [9]. In this context, mathematical models will be useful to describe the complex dynamics of the neural interactions and to orient the data analysis procedures of the large amount of data that will be obtained from the multielectrode recordings.

\section{CONCLUSION}

Our experiments and the results we obtained are preliminary and the most important work will be related to multielectrode recordings, mathematical modeling of the neural activity and the possibility of designing silicon-nerve interfaces that will adapt the sensory submodality of the connections to the expected by the target neurons. Finally we have to underline that the principal questions related to the development of artificial prostheses, that means the working principles of the information processing in the lower relay stations of the somatosensory pathway have not yet been studied.

\section{REFERENCES}

[1] E.P. Gardner, C.I. Palmer, H.A. Hämäläinen, and S. Warren, "On certain integrals of Lipschitz-Hankel type involving products of Bessel functions," J.Neurophysiol., pp. 6737-6772, 1992.

[2] J.J. Hopfield, "Pattern recognition computation using action potential timing for stimulus representation," Nature, vol. 376, pp.33-36, 1995.

[3] E.R. Kandel, J.H. Schwartz, and T.M. Jessell, Principles of neural science, 3 rd ed New York: McGraw-Hill, 2000.

[4] http://www.cordis.lu/ist/fetni-sy.htm
[5] G. Laurent, "Olfactory processing: maps, time and codes," Curr.opin.neurobiol., vol. 7, pp. 547-553, 1997.

[6] F. Panetsos, A. Núñez, and C. Avendaño, C. "Sensory information processing in the dorsal column nuclei by neuronal oscillators," Neuroscience, vol. 84, pp.635-639, 1998.

[7] W. Singer, and C.M. Gray, "Visual feature integration and the temporal correlation hypothesis". Annu. Rev. Neurosci., vol. 18, pp.555-586, 1995.

[8] T. Stieglitz, H.H. Ruf, M. Gross, M. Schuettler, and J.-U. Meyer, "A biohybrid system to interface peripheral nerves after traumatic lesions: design of a high channel sieve electrode," Biosensors and Bioelectronics, vol. 17, pp.685696,2002

[9] F. Valverde, "The pyramidal tract in rodents. A study of its relations with the posterior column nuclei, dorsolateral reticular formation of the medulla oblongata, and cervical spinal cord," Z.Zellforsch., vol. 71, pp.297-363, 1966. 